Pacific Journal of Mathematics

OSCILLATION AND NONOSCILLATION CRITERIA FOR SOM
SELF-ADJOINT EVEN ORDER LINEAR DIFFERENTIAL

ROGER T. LEWIS 


\title{
OSCILLATION AND NONOSCILLATION CRITERIA FOR SOME SELF-ADJOINT EVEN ORDER LINEAR DIFFERENTIAL OPERATORS
}

\author{
ROGER T. LEWIS
}

\begin{abstract}
Oscillation and nonoscillation results are presented for the operator

$$
L_{2 n} y=\sum_{k=0}^{n}(-1)^{n-k}\left(p_{k} y^{(n-k)}\right)^{(n-k)}
$$

where $p_{0}(x)>0$ on $(0, \infty)$ and for $k=0,1, \cdots, n, p_{k}$ is a realvalued, $n-k$ times differentiable function on $(0, \infty)$. Also, $y$ is an element of the set of all real-valued, $2 n$ - fold continuously differentiable, finite functions on $(0, \infty)$.

In particular, a nonoscillation result is given for $L_{2 n}$ without sign restrictions on the coefficients. Oscillation results are given for $L_{4}$ without the requirement that $p_{1}$ be negative for large $x$. Finally, the oscillation of
\end{abstract}

$$
L_{2 n} y=(-1)^{n}\left(r y^{(n)}\right)^{(n)}+p y
$$

is considered for $r(x)$ not necessarily bounded.

The oscillatory behavior of $L_{4}$ has been considered by Leighton and Nehari [8], Barrett [1], and Hinton [4]. In general, $L_{2 n}$ has been considered by Glazman [2], Hinton [5], Hunt [6], and Hunt and Namboodiri [7].

DEFINITION 0.1. The operator $L_{2 n}$ is called oscillatory on $[a, b]$ provided there is a function $y, y \neq \equiv 0$, and numbers $c$ and $d$ for which $a \leqq c<d \leqq b$ such that $L_{2 n} y=0$ and

$$
y^{(k)}(c)=0=y^{(k)}(d) \text { for } k=0,1, \cdots, n-1 .
$$

Otherwise, $L_{2 n}$ is called nonoscillatory on $[a, b]$. The operator $L_{2 n}$ is called oscillatory on $[a, \infty)$ if for any given $c \geqq a$ there is a $d>c$ such that $L_{2 n}$ is oscillatory on $[c, d]$.

Definition 0.2. Given a positive integer $n$ and a number $a$ define $\mathfrak{D}_{n}(b)$ for all $b>a$ to be the set of all real-valued functions $y$ with the following properties:

(a) $y^{(k)}$ is absolutely continuous on $[a, b]$ for $k=0,1, \cdots, n-1$,

(b) $y^{(n)}$ is essentially bounded on $[a, b]$, and

(c) $y^{(k)}(a)=0=y^{(k)}(b)$ for $k=0,1, \cdots, n-1$.

For $y \in \mathfrak{D}_{n}(b)$ define

$$
I_{b}(y)=\int_{a}^{b} \sum_{k=0}^{n} p_{k}(x)\left(y^{(n-k)}(x)\right)^{2} d x
$$


which is called the quadratic functional for $L_{2 n}$.

The following theorem has provided the primary motivation for the results which are to follow.

THEOREM 0.1 (Reid [9]). The following two statements are equivalent.

(i) The operator $L_{2 n}$ is nonoscillatory on $[a, b]$.

(ii) If $y \in \mathfrak{D}_{n}(b)$ and $y \not \equiv 0$ then $I_{b}(y)>0$.

Consequently, in order to show that $L_{2 n}$ is oscillatory on $(0, \infty)$, given any $a>0$, it will suffice to construct a $y \in \mathscr{D}_{n}(b)$ for some $b>$ $a$ for which $I_{b}(y)$ is not positive and $y \not \equiv 0$. This is the technique of proof for all of the oscillation theorems which follow.

This method of proof is especially conducive to oscillation theorems which require that integral conditions be met by the coefficients of $L_{2 n}$. For example, Glazman [2, p. 104] showed that $(-1)^{n} y^{(2 n)}+p y$ is oscillatory on $(0, \infty)$ when $\int^{\infty} p=-\infty$ (see Theorem 3.2).

Initially, the construction of $y$ is suggested by the conditions of the hypothesis on the coefficients of $L_{2 n}$ and the corresponding quadratic formula. For example, to establish the above result, Glazman let $y \equiv 1$ over the major portion of the interval $[a, b]$. To show that $y^{i v}-\left(q y^{\prime}\right)^{\prime}$ is oscillatory when $\int^{\infty} q=-\infty$ (see Theorem 2.2) the author let $y(x)=x-a$ over a portion of $[a, b]$. Next, we construct $y$ over the remaining portion of $[a, b]$ to insure that $y \in \mathfrak{D}_{n}(b)$ and the integral of $p_{n-k} \cdot y^{(n-k)^{2}}$ is bounded above for $k=0,1, \cdots, n$ independent of $b$.

For other proofs using this method the reader should consult Glazman [2, pp. 95-105] and Hinton [4].

1. The nonoscillation of $L_{2 n}$.

LEMMA 1.1 (Glazman [2, p. 83]).

(i) If $g(a)=0$ for some $a>0$ and $g^{\prime}$ is continuous on $[a, b]$, then

$$
\int_{a}^{b} x^{-2 m}(g(x))^{2} d x \leqq\left(\frac{2}{2 m-1}\right)^{2} \int_{a}^{b} x^{-2 m+2}\left(g^{\prime}(x)\right)^{2} d x
$$

for $m$ a positive integer. Moreover, if $g \not \equiv 0$ on $[a, b]$, the above inequality is strict. then

(ii) If $g \not \equiv 0, g^{(m)}$ is continuous on $[a, b]$, and $g(a)=\cdots=g^{(m-1)}(a)=0$,

$$
\int_{a}^{b} x^{-2 m}(g(x))^{2} d x<\left(\frac{2^{m}}{1 \cdot 3 \cdot \cdots \cdot(2 m-1)}\right)^{2} \int_{a}^{b}\left(g^{(m)}\right)^{2} d x
$$


A well known result in oscillation theory is a sufficient condition for the nonoscillation of $L_{2}$ due to Hille [3]. A generalization of this result for $L_{2 n}$ is given in the next theorem.

THeORem 1.1. For $L_{2 n}$ defined above with $p_{0}(x) \equiv 1$ let $P_{k}^{0}(x)=$ $p_{k}(x)$ and

$$
P_{k}^{m}(x)=\int_{x}^{\infty} P_{k}^{m-1}(t) d t
$$

for $m$ an integer greater than or equal to one when

$$
-\infty<\int^{\infty} P_{k}^{m-1}(t) d t<\infty .
$$

If for $k=1, \cdots, n$ and $x \geqq a$ we have $-\infty<\int_{a}^{\infty} P_{k}^{m}<\infty$ for $m=0,1, \cdots, \quad k-1, \quad x^{k}\left|P_{k}^{k}\right| \leqq a_{k}$, and $\sum_{k=1}^{n} a_{k} M_{k} \leqq 1$ where $M_{k}=k ! 2^{4 k-1} /(2 k) !$, then $L_{2 n}$ is nonoscillatory on $[a, b]$ for all $b>a$.

Proof. The proof is given only for $n>1$. Suppose $L_{2 n}$ is oscillatory on $[a, b]$. Then, there are numbers $c$ and $d$ and a function $y$ which is not identically zero such that $L_{2 n} y=0$ and $y^{(k)}(c)=0=$ $y^{(k)}(d)$ for $k=0,1, \cdots, n-1$. Since $\left(L_{2 n} y\right) y=0$ then

$$
-\sum_{k=1}^{n}(-1)^{n-k} \int_{c}^{d}\left(p_{k} y^{(n-k)}\right)^{(n-k)} y=(-1)^{n} \int_{c}^{d} y^{(2 n)} y=\int_{c}^{d}\left[y^{(n)}\right]^{2},
$$

by integrating by parts $n$ times. By integrating by parts $n-k$ times we find that

$$
-\int_{c}^{d}(-1)^{n-k}\left(p_{k} y^{(n-k)}\right)^{(n-k)} y=-\int_{c}^{d} p_{k}\left(y^{(n-k)}\right)^{2} .
$$

However, by integrating by parts $k$ times and using Leibniz's rule we obtain

$$
\begin{aligned}
-\int_{c}^{d} p_{k}\left(y^{(n-k)}\right)^{2}=-\int_{c}^{d} P_{k}^{k}\left[\left(y^{(n-k)}\right)^{2}\right]^{(k)}=-\int_{c}^{d} P_{k}^{k} \cdot \sum_{i=0}^{k}\left(\begin{array}{c}
k \\
i
\end{array}\right)\left(y^{(n-k)}\right)^{(k-i)}\left(y^{(n-k)}\right)^{(i)} \\
\quad \leqq \sum_{i=0}^{k}\left(\begin{array}{c}
k \\
i
\end{array}\right) \int_{c}^{d} \frac{\left|y^{(n-i)}\right|}{t^{i}} \cdot \frac{\left|y^{(n-k+i)}\right|}{t^{k-i}} \cdot t^{k}\left|P_{k}^{k}\right| \\
\leqq a_{k} \sum_{i=0}^{k}\left(\begin{array}{c}
k \\
i
\end{array}\right) \cdot \int_{c}^{d} \frac{\left|y^{(n-i)}\right|}{t^{k}} \cdot \frac{\left|y^{(n-k+i)}\right|}{t^{k-i}} \\
\quad=a_{k}\left[2 \int_{c}^{d}\left|y^{(n)}\right| \cdot \frac{\left|y^{(n-k)}\right|}{t^{k}}+\sum_{i=1}^{k-1}\left(\begin{array}{c}
k \\
i
\end{array}\right) \int_{c}^{d} \frac{\left|y^{(n-i)}\right|}{t^{i}} \cdot \frac{\left|y^{(n-k+i)}\right|}{t^{k-i}}\right] \\
\leqq a_{k}\left[2\left\|y^{(n)}\right\|\left\|_{2}\right\| \frac{y^{(n-k)}}{t^{k}}\left\|_{2}+\sum_{i=1}^{k-1}\left(\begin{array}{c}
k \\
i
\end{array}\right)\right\| \frac{y^{(n-i)}}{t^{i}}\left\|_{2}\right\| \frac{y^{(n-k+i)}}{t^{k-i}} \|_{2}\right]
\end{aligned}
$$




$$
\begin{aligned}
& <a_{k}\left[\left\|y^{(n)}\right\|_{2}^{2}\left(\frac{2^{k+1}}{1 \cdot 3 \cdot \cdots \cdot(2 k-1)}\right)\right. \\
& \left.+\sum_{i=1}^{k-1}\left(\begin{array}{c}
k \\
i
\end{array}\right)\left\|y^{(n)}\right\|_{2}^{2}\left(\frac{2^{i}}{1 \cdot 3 \cdot \cdots \cdot(2 i-1)}\right)\left(\frac{2^{k-i}}{1 \cdot 3 \cdot \cdots \cdot(2(k-i)-1)}\right)\right] \\
& =a_{k} C_{k} \int_{c}^{d}\left[y^{(n)}\right]^{2}
\end{aligned}
$$

by Lemma 1.1 and the Cauchy inequality where

$$
\begin{aligned}
C_{k}= & \frac{2^{k+1}}{1 \cdot 3 \cdot \cdots \cdot(2 k-1)} \\
& +\sum_{i=1}^{k-1}\left(\begin{array}{l}
k \\
i
\end{array}\right) \cdot\left(\frac{2^{k}}{[1 \cdot 3 \cdot \cdots \cdot(2 i-1)][1 \cdot 3 \cdots(2(k-i)-1)]}\right) .
\end{aligned}
$$

A simplification shows that

$$
C_{k}=\left[2^{2 k} k ! /(2 k) !\right] \sum_{i=0}^{k}\left(\begin{array}{l}
2 k \\
2 i
\end{array}\right) .
$$

Since

$$
0=(1-1)^{2 k}=\sum_{i=0}^{2 k}(-1)^{i}\left(\begin{array}{c}
2 k \\
i
\end{array}\right)=\sum_{i=0}^{k}\left(\begin{array}{l}
2 k \\
2 i
\end{array}\right)-\sum_{i=1}^{k}\left(\begin{array}{c}
2 k \\
2 i-1
\end{array}\right),
$$

then

$$
2^{2 k-1}=\frac{1}{2}(1+1)^{2 k}=\frac{1}{2}\left[\sum_{i=0}^{k}\left(\begin{array}{l}
2 k \\
2 i
\end{array}\right)+\sum_{i=1}^{k}\left(\begin{array}{c}
2 k \\
2 i-1
\end{array}\right)\right]=\sum_{i=0}^{k}\left(\begin{array}{l}
2 k \\
2 i
\end{array}\right) .
$$

Therefore,

$$
C_{k}=\left[2^{4 k-1} k !\right] /(2 k) !=M_{k} \text {. }
$$

Consequently,

$$
\begin{aligned}
\int_{c}^{d}\left[y^{(n)}\right]^{2} & =-\sum_{k=1}^{n}(-1)^{n-k} \int_{c}^{d}\left(p_{k} y^{(n-k)}\right)^{(n-k)} y \\
& =-\sum_{k=1}^{n} \int_{c}^{d} p_{k}\left(y^{(n-k)}\right)^{2}<\sum_{k=1}^{n} a_{k} M_{k} \int_{c}^{d}\left[y^{(n)}\right]^{2} \leqq \int_{c}^{d}\left[y^{(n)}\right]^{2}
\end{aligned}
$$

which is a contradiction. Therefore, $L_{2 n}$ is nonoscillatory on $[a, b]$.

It will be useful in applying Theorem 1.1 to note that $M_{k+1}=$ $8 M_{k} /(2 k+1)$.

For the remainder of this paper we will assume that $p_{k}(x)$ is identically zero for $k=1$ to $n-2$ and will denote $p_{0}(x), p_{n-1}(x)$, and $p_{n}(x)$ by $r(x), q(x)$, and $p(x)$ respectively. Similarly, $P_{n}^{k}(x)$ and $P_{n-1}^{k}(x)$ will be denoted by $P_{k}(x)$ and $Q_{k}(x)$ respectively.

If $p(x)=k x^{-4}, r \equiv 1$, and $q \equiv 0$, then $L_{4} y=0$ is the familiar Euler equation. In this case, $L_{4}$ is oscillatory if and only if $k<-9 / 16$. 
Also, $k<-9 / 16$ and $p(x)=k x^{-4}$ implies $x^{2} P_{2}(x)<-3 / 32$. Theorem 1.1 shows that $L_{4} y=y^{i v}+p y$ is nonoscillatory when $x^{2}\left|P_{2}(x)\right| \leqq 3 / 32$.

2. The oscillation of $L_{4}$. Using Theorem 0.1, Hinton [4] showed that $L_{4}$ is oscillatory when $\int^{\infty} 1 / r=\infty, q \leqq 0$, and $\int^{\infty} p=-\infty$. The same technique yields the following results.

THEOREM 2.1. Suppose that $r(x) \leqq N, q(x) \leqq M$ and $\int^{\infty} p=-\infty$ for $x>0$, then $L_{4} y=\left(r y^{\prime \prime}\right)^{\prime \prime}-\left(q y^{\prime}\right)^{\prime}+p y$ is oscillatory on $(0, \infty)$.

THEOREM 2.2. If $0<r(x) \leqq M, \int^{\infty} q=-\infty$, and $\int^{\infty} x^{2}|p(x)|<\infty$ then $L_{4} y=\left(r y^{\prime \prime}\right)^{\prime \prime}-\left(q y^{\prime}\right)^{\prime}+p y$ is oscillatory on $(0, \infty)$.

Proof. Let $\xi(x)=x^{2} / 2$. Define $y(x)$ as follows:

$$
y(x)= \begin{cases}0 & x<a \\ \xi(x-a) & a \leqq x<a+1 \\ x-a-1 / 2 & a+1 \leqq x<b_{1} \\ -\xi\left(x-b_{2}\right)+b_{1}-a & b_{1} \leqq x<b_{2}=b_{1}+1 \\ b_{1}-a & b_{2} \leqq x<b_{3} \\ -\xi\left(x-b_{3}\right)+b_{1}-a & b_{3} \leqq x<b_{4} \\ -\xi^{\prime}\left(b_{4}-b_{3}\right)\left(x-b_{4}\right)+b_{1}-a-\xi\left(b_{4}-b_{3}\right) & b_{4} \leqq x<b_{5} \\ \xi(x-b) & b_{5} \leqq x<b \\ 0 & b \leqq x .\end{cases}
$$

It is easy to show that

$$
\int_{a}^{d} r\left(y^{\prime \prime}\right)^{2}+p y^{2}<16 M+\int_{a}^{\infty} x^{2}|p|
$$

if we require that $b_{4}-b_{3}=b-b_{5} \leqq 1$. There is a number $c$ such that

$$
1+16 M+\int_{a}^{\infty} x^{2}|p|+\int_{a}^{a+1} q\left(y^{\prime}\right)^{2}+\int_{a+1}^{x} q \leqq 0
$$

for all $x \geqq c$.

Let $Y_{1}(x)=\int_{c}^{x} q(t) d t$. Since $Y_{1}(x)$ tends to $-\infty$ as $x$ tends to $c$ there is a number $b_{1}$ which is the last zero of $Y_{1}(x)$. Hence,

$$
\int_{b_{1}}^{b_{2}} q\left(y^{\prime}\right)^{2}=\left.Y_{1}(x)\left(y^{\prime}\right)^{2}\right|_{b_{1}} ^{b_{2}}-2 \int_{b_{1}}^{b_{2}} y^{\prime} y^{\prime \prime} Y_{1}<0
$$

since $Y_{1}\left(b_{1}\right)=0=y^{\prime}\left(b_{2}\right), y^{\prime \prime}=-1, y^{\prime} \geqq 0$, and $Y_{1}<0$ on $\left(b_{1}, b_{2}\right]$.

Let $Y_{2}(x)=\int_{b_{2}}^{x} q(t) d t$ and let $b_{3}$ be the last zero of $Y_{2}$. Pick $b_{4}$ so 
that $-1 / 2 \leqq Y_{2}(x) \leqq 0$ on $\left[b_{3}, b_{4}\right]$ and $b_{4}-b_{3} \leqq 1$. Since $y^{\prime}=0$ on $\left[b_{2}, b_{3}\right],-1 \leqq y^{\prime} \leqq 0$ on $\left[b_{3}, b\right], Y_{2} \leqq 0$ on $\left[b_{3}, \infty\right), \int_{b_{1}}^{b_{2}} q\left(y^{\prime}\right)^{2}<0$ we have that

$$
\begin{aligned}
\int_{b_{1}}^{b} q\left(y^{\prime}\right)^{2} & <\int_{b_{3}}^{b} q\left(y^{\prime}\right)^{2}=\left.Y_{2}(x)\left(y^{\prime}\right)^{2}\right|_{b_{3}} ^{b}-2 \int_{b_{3}}^{b} y^{\prime} y^{\prime \prime} Y_{2} \\
& =-2 \int_{b_{3}}^{b_{4}} y^{\prime}(-1) Y_{2}-2 \int_{b_{5}}^{b} y^{\prime}(1) Y_{2} \\
& <2 \int_{b_{3}}^{b_{4}} y^{\prime} Y_{2}<2 \int_{b_{3}}^{b_{4}}\left|y^{\prime} Y_{2}\right|<1 .
\end{aligned}
$$

Consequently,

$$
\begin{aligned}
I_{b}(y) & =\int_{a}^{b} r\left(y^{\prime \prime}\right)^{2}+q\left(y^{\prime}\right)^{2}+p y^{2} \\
& <16 M+\int_{a}^{\infty} x^{2}|p|+\int_{a}^{a+1} q\left(y^{\prime}\right)^{2}+\int_{a+1}^{b_{1}} q+1 \leqq 0
\end{aligned}
$$

which completes the proof.

We now know that $L_{4}$ is oscillatory on $(0, \infty)$ is for $r$ bounded either $\int^{\infty} p=-\infty$ and $q \leqq 0$ or $\int^{\infty} q=-\infty$ and $p \leqq 0$. These facts suggest the results of the following theorem.

Theorem 2.3. If $\int^{\infty} p=-\infty, \int^{\infty} q=-\infty$, and $0<r(x) \leqq M$ then $L_{4}$ is oscillatory on $(0, \infty)$.

Proof. Except for some changes in the parameters we may define $y(x)$ as in the proof of Theorem 2.2. As before, if $b_{4}-b_{3}=b_{6}-b_{5} \leqq$ 1 then $\int_{a}^{b} r\left(y^{\prime \prime}\right)^{2} \leqq 16 M$.

There is a number $c$ such that

$$
1+16 M+\int_{a}^{a+1} q\left(y^{\prime}\right)^{2}+\int_{a+1}^{x} q<0
$$

for all $x \geqq c$. Let $Y(x)=\int_{c}^{x} q(t) d t$ and let $b_{1}$ be the last zero of $Y(x)$. Integrating by parts we obtain the fact that

$$
\int_{b_{1}}^{b} q\left(y^{\prime}\right)^{2}=-2 \int_{b_{1}}^{b} y^{\prime} y^{\prime \prime} Y
$$

since $Y\left(b_{1}\right)=0=y^{\prime}(b)$. Since $y^{\prime} \geqq 0, y^{\prime \prime}=-1$, and $Y \leqq 0$ on $\left[b_{1}, b_{2}\right]$ then

$$
-2 \int_{b_{1}}^{b} y^{\prime} y^{\prime \prime} Y<-2 \int_{b_{2}}^{b} y^{\prime} y^{\prime \prime} Y
$$

Since $y^{\prime \prime}=0$ on $\left[b_{2}, b_{3}\right]$ and $\left[b_{4}, b_{5}\right], y^{\prime} \leqq 0$ on $\left[b_{5}, b\right], y^{\prime \prime}=1$ on $\left[b_{5}, b\right]$, and $Y<0$ on $\left[b_{5}, b\right]$, then 


$$
-2 \int_{b_{2}}^{b} y^{\prime} y^{\prime \prime} Y<-2 \int_{b_{3}}^{b_{4}} y^{\prime} y^{\prime \prime} Y=2 \int_{b_{3}}^{b_{4}} y^{\prime} Y
$$

But, on $\left[b_{3}, b_{4}\right], y^{\prime} \leqq 1$. Consequently,

$$
\int_{b_{1}}^{b} q\left(y^{\prime}\right)^{2}<2 \int_{b_{3}}^{b_{4}} y^{\prime} Y \leqq 2 \int_{b_{3}}^{b_{4}}|Y|
$$

Since $\int^{\infty} p=-\infty$, there is a number $d>b_{2}$ such that for $x \geqq d$

$$
\int_{a}^{b_{2}} p y^{2}+\left(b_{1}-a\right)^{2} \int_{b_{2}}^{x} p<0 .
$$

Let $W(x)=\int_{d}^{x} p$ and $b_{3} \geqq d$ be the last zero of $W$. Hence,

$$
\int_{b_{3}}^{b} p y^{2}=-2 \int_{b_{3}}^{b} y y^{\prime} W(t) d t<0 .
$$

Let $N=\max \left\{|Y(x)|: x \in\left[b_{3}, b_{3}+1\right]\right\}$ which we may assume is greater than or equal to one. Pick $b_{4}$ so that $b_{4}-b_{3}=1 /(2 N)$. Consequently,

$$
\int_{b_{1}}^{b} q\left(y^{\prime}\right)^{2}<1
$$

Pick $b_{5}$ so that $\lim _{x \rightarrow b_{5}}-y(x)=\left(b_{4}-b_{3}\right)^{2} / 2$ and pick $b$ so that $b-b_{5}=$ $b_{4}-b_{3}$. We now have that

$$
I_{b}(y)<16 M+\int_{a}^{a+1} q\left(y^{\prime}\right)^{2}+\int_{a+1}^{x} q+1+\int_{a}^{b_{2}} p y^{2}+\int_{b_{2}}^{b_{3}}\left(b_{1}-a\right)^{2} p<0 .
$$

THEOREM 2.4. If $0<r(x) \leqq M,-\infty<\int^{\infty} p<\infty, \int^{\infty} P_{1}=-\infty$, $\int^{\infty}|q| x^{-1}<\infty$, and $q(x) \rightarrow 0$ as $x \rightarrow \infty$ then $L_{4}$ is oscillatory on $(0, \infty)$.

Proof. Let $\xi(x)=-\left(3 x^{3}-5 x^{2}\right) / 2, \alpha(x)=\sqrt{x}$, and $\beta(x)=x^{2}$. Let

$$
y(x)= \begin{cases}0 & x<a \\ \xi(x-a) & a \leqq x<a+1 \\ \alpha(x-a) & a+1 \leqq x<b_{1} \\ -\beta\left(x-b_{2}\right)+\alpha\left(b_{1}-a\right)+\beta\left(b_{1}-b_{2}\right) & b_{1} \leqq x<b_{2} \\ \alpha\left(b_{1}-a\right)+\beta\left(b_{1}-b_{2}\right) & b_{2} \leqq x<b_{3} \\ -\beta\left(x-b_{3}\right)+y\left(b_{2}\right) & b_{3} \leqq x<b_{4} \\ \alpha(b-x) & b_{4} \leqq x<b_{5} \\ \xi(b-x) & b_{5} \leqq x<b \\ 0 & b \leqq x .\end{cases}
$$


Given $b_{1}$ and $b_{3}$ we choose $b_{4}, b_{5}$, and $b$ so that $b_{4}-b_{3}=b_{2}-b_{1}, b_{5}-$ $b_{4}=b_{1}-(a+1)$, and $b=b_{5}+1$. Actually, only $b_{1}$ and $b_{3}$ will be chosen for reasons other than symmetry and the continuity of $y$ and $y^{\prime}$.

First, note that since we are going to pick $b_{1}, \cdots, b_{5}$ so that $y \in \mathfrak{D}_{2}(b)$ then

$$
\int_{a}^{b} p y^{2}=-\left.P_{1} y^{2}\right|_{a} ^{b}+\int_{a}^{b} P_{1}\left(y^{2}\right)^{\prime}=\int_{a}^{b} P_{1}\left(y^{2}\right)^{\prime} .
$$

Hence,

$$
I_{b}(y) \leqq \int_{a}^{b} M\left(y^{\prime \prime}\right)^{2}+q\left(y^{\prime}\right)^{2}+P_{1}\left(y^{2}\right)^{\prime}
$$

Calculations show that

$$
\int_{a}^{b} M\left(y^{\prime \prime}\right)^{2} \leqq M\left[2 \int_{0}^{1}(5-9 x)^{2}+2+\frac{1}{2} \int_{1}^{\infty} x^{-3} d x\right] \equiv M_{1}
$$

since $y^{\prime}$ being continuous requires that $0<b_{2}-b_{1}=b_{4}-b_{3} \leqq 1 / 4$.

Since $\lim _{x \rightarrow \infty} q(x)=0$ and $q$ is continuous then $q$ is bounded by some number, $B$, on $[a, \infty)$. Let

$$
A=4 B \int_{0}^{1} u^{2}+B \int_{0}^{1}\left(-\frac{9}{2} u^{2}+5 u\right)^{2} d u+1 .
$$

There is a number $c$ so that

$$
\begin{aligned}
M_{1} & +2+\int_{a}^{a+1}\left[q\left(y^{\prime}\right)^{2}+P_{1}\left(y^{2}\right)^{\prime}\right] \\
& +A+B+(a+1) \int_{a+1}^{\infty} x^{-1}|q(x)| \leqq-\int_{a+1}^{x} P_{1}(t) d t
\end{aligned}
$$

and $\left|P_{1}(x)\right| \leqq 1$ for all $x \geqq c$ since $P_{1} \rightarrow 0$ as $x \rightarrow \infty$.

Let $R(x)=\int_{c}^{x} P_{1}(t) d t$ and $b_{1}$ be the last zero of $R(x)$. Pick $b_{2}$ so that $1 /\left(2 \sqrt{b_{1}-a}\right)=-2\left(b_{1}-b_{2}\right)$ which insures that $y^{\prime}$ is continuous at $b_{1}$. We now have that

$$
\begin{aligned}
\int_{a+1}^{b_{1}} q\left(y^{\prime}\right)^{2} & \leqq \int_{a+1}^{b_{1}}(x-a)^{-1}|q|=\int_{a+1}^{b_{1}} x(x-a)^{-1} x^{-1}|q| \\
& <(a+1) \int_{a+1}^{b_{1}} x^{-1}|q|<(a+1) \int_{a+1}^{\infty} x^{-1}|q|
\end{aligned}
$$

and

$$
\int_{b_{1}}^{b_{2}} q\left(y^{\prime}\right)^{2} \leqq B \int_{b_{1}}^{b_{2}} 4\left(x-b_{2}\right)^{2}<B
$$

since $b_{2}-b_{1} \leqq 1 / 4$. Also, 


$$
\begin{aligned}
\int_{b_{1}}^{b_{2}} P_{1}\left(y^{2}\right)^{\prime} & =\left.2 y y^{\prime} R\right|_{b_{1}} ^{b_{2}}-2 \int_{b_{1}}^{b_{2}}\left[\left(y^{\prime}\right)^{2}+y y^{\prime \prime}\right] R \leqq-2 \int_{b_{1}}^{b_{2}}\left(y^{\prime}\right)^{2} R \\
& =-2\left[\left.R(t) \int_{b_{2}}^{t}\left(y^{\prime}\right)^{2}\right|_{b_{1}} ^{b_{2}}-\int_{b_{1}}^{b_{2}}\left(P_{1}(t) \int_{b_{2}}^{t}\left(y^{\prime}\right)^{2}\right)\right] \\
& \leqq 2 \int_{b_{1}}^{b_{2}}\left|P_{1}(t)\right| \int_{b_{1}}^{b_{2}}\left(y^{\prime}\right)^{2}<1
\end{aligned}
$$

since $y^{\prime}\left(b_{2}\right)=0=R\left(b_{1}\right), y^{\prime \prime} \leqq 0, y \geqq 0$, and $R \leqq 0$ on $\left[b_{1}, b_{2}\right]$. Pick $b_{3}$ so that $\left|P_{1}(x)\right| \leqq\left[6 y\left(b_{2}\right)\left(b_{2}-a\right)\right]^{-1}$ and $|q(x)| \leqq 4\left(b_{1}-a-1\right)^{-1}$ for $x \geqq$ $b_{3}$. Consequently,

$$
\int_{b_{2}}^{b} P_{1}\left(y^{2}\right)^{\prime}=\int_{b_{3}}^{b} P_{1}\left(y^{2}\right)^{\prime} \leqq 2 \int_{b_{3}}^{b}\left|P_{1}\|y\| y^{\prime}\right| \leqq 1
$$

since $|y| \leqq y\left(b_{2}\right),\left|y^{\prime}\right| \leqq 3$, and $b-b_{3}=b_{2}-a$. Also,

$$
\begin{aligned}
\int_{b_{2}}^{b} q\left(y^{\prime}\right)^{2} \leqq & B \int_{b_{3}}^{b_{4}} 4\left(x-b_{3}\right)^{2}+(1 / 4) \int_{b_{4}}^{b_{5}}\left(b_{6}-x\right)^{-1}|q(x)| \\
& +B \int_{b_{5}}^{b}\left[5\left(b_{6}-x\right)-9\left(b_{6}-x\right)^{2} / 2\right]^{2} \\
< & (A-1)+(1 / 4) \int_{b_{4}}^{b_{5}}|q| \leqq A
\end{aligned}
$$

In conclusion,

$$
\begin{gathered}
I_{b}(y) \leqq M_{1}+\int_{a}^{a+1} q\left(y^{\prime}\right)^{2}+(a+1) \int_{a+1}^{\infty} x^{-1}|q|+B+A \\
+\int_{a}^{a+1} P_{1}\left(y^{2}\right)^{\prime}+\int_{a+1}^{b_{1}} P_{1}+1+1 \leqq 0 .
\end{gathered}
$$

The conditions of Theorem 2.4, $\int^{\infty}|q| / x<\infty$ and $\lim _{x \rightarrow \infty} q(x)=0$, could be replaced by the conditions, $\int^{\infty}|q|<\infty$ and $q$ bounded, to obtain the same result with a similar proof.

ThEOREM 2.5. Suppose $0<r(x) \leqq M, \int^{\infty} p<\infty, \int^{\infty} P_{1}<\infty$, and $P_{1}(x) \leqq C x^{-4}$ for $x>0$. If $\lim _{x \rightarrow \infty} \inf x^{2} P_{2}(x)<-7 \frac{1}{32} M$ then $L_{4} y=$ $\left(r y^{\prime \prime}\right)^{\prime \prime}+p y$ is oscillatory on $(0, \infty)$.

Proof. We will use the fact that for $a>0$

$$
I_{b}(y)=\int_{a}^{b}\left[r\left(y^{\prime \prime}\right)^{2}+2 y y^{\prime} P_{1}\right]
$$

for $y$ given below. Let $\xi(x)=-\left(3 x^{3}-5 x^{2}\right) / 2, \alpha(x)=\sqrt{x}$, and $\beta(x)=$ $x^{2}$. For $0<\mu<1,0<\sigma \leqq 1, \rho>0$, and $0<\gamma \leqq 1$ define $y$ as follows: 


$$
y(x)= \begin{cases}0 & x<\mu \rho \\ \xi\left(\frac{x-\mu \rho}{\rho[1-\mu]}\right) & \mu \rho \leqq x<\rho \\ \alpha\left(\frac{x-\mu \rho}{\rho[1-\mu]}\right) & \rho \leqq x<R \\ -\beta(x-(R+\sigma))+\beta(\sigma)+\alpha\left(\frac{R-\mu \rho}{\rho[1-\mu]}\right) & R \leqq x<R+\sigma \\ \beta(\sigma)+\alpha\left(\frac{R-\mu \rho}{\rho[1-\mu]}\right) & R+\sigma \leqq x<N \\ -\beta(x-N)+y(R+\sigma) & N \leqq x<N+\gamma \\ -2 \gamma(x-N-\gamma)+y(R+\sigma)-\beta(\gamma) & N+\gamma \leqq x<b-\gamma \\ \beta(x-b) & b-\gamma \leqq x<b \\ 0 & b \leqq x .\end{cases}
$$

Calculations show that

$$
\begin{aligned}
& \int_{\mu \rho}^{b} r\left(y^{\prime \prime}\right)^{2} \\
& \quad<\left(1 /\left[\rho^{3}(1-\mu)\right]\right)\left[7 \frac{1}{32} M(1-\mu)^{-2}+4 \sigma M \rho^{3}(1-\mu)+8 \gamma M \rho^{3}(1-\mu)\right] .
\end{aligned}
$$

Since

$$
\lim \inf x^{2} \int_{x}^{\infty} P_{1}<-7 \frac{1}{32} M,
$$

there is a $\delta>0$ and a sequence $\left\langle\rho_{k}\right\rangle \rightarrow \infty$ for which

$$
\lim _{k \rightarrow \infty} \rho_{k}^{2} \int_{\rho_{k}}^{\infty} P_{1} \leqq-\left(7 \frac{1}{32} M+2 \delta\right) .
$$

Pick $\mu$ so close to zero that $7(1 / 32) M(1-\mu)^{-2}=7(1 / 32) M+7 \delta / 8$. There is a positive integer $N$ so large that $\mu \rho_{k}>a, C\left(\mu^{-3}-1\right) / \rho_{k}<$ $\delta / 8$, and

$$
\rho_{k}^{2} \int_{\rho_{k}}^{\infty} P_{1} \leqq-\left(7 \frac{1}{32} M+7 \delta / 4\right)
$$

for all $k \geqq N$. Let $\rho=\rho_{N}$.

Given $R$, we will pick $\sigma$ so that $y^{\prime}(x)$ is continuous at $x=R$. Therefore,

$$
\sigma=1 /(4 \sqrt{\rho[1-\mu](R-\mu \rho)}) .
$$

Since $\sigma \rightarrow 0$ as $R \rightarrow \infty$ and $P_{1}$ is bounded on $[a, \infty)$ pick $R$ so large that

$$
\rho^{2} \int_{\rho}^{R} P_{1} \leqq-\left(7 \frac{1}{32} M+13 \delta / 8\right),
$$




$$
\sigma<(\delta / 8) /\left[4 M \rho^{3}(1-\mu)\right],
$$

and $\sigma\left|P_{1}\right|<\delta /\left(8 \rho^{2}\right)$ for all $x \geqq R$. On $[R, R+\sigma]$,

$$
0<y(x) \leqq \alpha((x-\mu \rho) /(\rho[1-\mu]))
$$

and

$$
0 \leqq y^{\prime}(x) \leqq \alpha^{\prime}((x-\mu \rho) /(\rho[1-\mu])) \cdot 1 /(\rho[1-\mu])
$$

which implies that $0 \leqq 2 y y^{\prime} \leqq 1 /(\rho[1-\mu])$ on $[R, R+\sigma]$.

On $[\mu \rho, \rho] 0 \leqq 2 y y^{\prime}<3 /(\rho[1-\mu])$. Hence,

$$
\begin{aligned}
2 \int_{a}^{b} y y^{\prime} P_{1}< & 3(\rho[1-\mu])^{-1} \int_{\mu \rho}^{\rho} P_{1}^{+}+(\rho[1-\mu])^{-1} \int_{\rho}^{R} P_{1} \\
& +(\rho[1-\mu])^{-1} \int_{R}^{R+\sigma}\left|P_{1}\right|+2 \int_{N}^{b}\left|y y^{\prime}\right|\left|P_{1}\right| \\
< & 3 C(\rho[1-\mu])^{-1} \int_{\mu \rho}^{\rho} x^{-4} d x+(\rho[1-\mu])^{-1} \int_{\rho}^{R} P_{1} \\
& +(\delta / 8) /\left(\rho^{3}[1-\mu]\right)^{-1}+2 \int_{N}^{b}\left|y y^{\prime} \| P_{1}\right|
\end{aligned}
$$

where $P_{1}^{+}(x)=P_{1}(x)$ when $P_{1}(x) \geqq 0$ and zero otherwise.

On $[N, b] 0 \leqq y(x) \leqq y(R+\sigma)$ and $\left|y^{\prime}\right| \leqq 2 \gamma$. Since $y$ is linear on $[N+\gamma, b-\gamma]$ we have that

$$
\left[y(R+\sigma)-2 \gamma^{2}\right] /[b-N-2 \gamma]=2 \gamma
$$

or

$$
b-N=[y(R+\sigma)] /(2 \gamma)+\gamma .
$$

Since $P_{1}(x) \rightarrow 0$ as $x \rightarrow \infty$ we can pick $N$ so large that

$$
\left|P_{1}\right| \leqq(\delta / 8) /\left(2[y(R+\sigma)]^{2} \rho^{3}[1-\mu]\right)
$$

for all $x \geqq N$. Pick $\gamma$ so small that $2 \gamma^{2}[y(R+\sigma)]^{-1}<1$ and

$$
8 M \gamma \rho^{3}[1-\mu]<\delta / 8 \text {. }
$$

Pick $b$ so that

$$
\lim _{x \rightarrow(b-\gamma)^{-}} y(x)=\gamma^{2} .
$$

We now have that

$$
\begin{aligned}
2 \int_{N}^{b}\left|y y^{\prime} \| P_{1}\right| & \leqq 4 \gamma y(R+\sigma) \int_{N}^{b}\left|P_{1}\right| \\
& \leqq 2 \gamma(b-N) \cdot(\delta / 8) /\left(y(R+\sigma) \rho^{3}[1-\mu]\right) \\
& =2 \gamma([y(R+\sigma) /(2 \gamma)]+\gamma) \cdot(\delta / 8) /\left(y(R+\sigma) \rho^{3}[1-\mu]\right) \\
& =(\delta / 8) /\left(\rho^{3}[1-\mu]\right)+2 \gamma^{2}(\delta / 8) /\left(y(R+\sigma) \rho^{3}[1-\mu]\right) \\
& <(\delta / 4) /\left(\rho^{3}[1-\mu]\right) .
\end{aligned}
$$


Consequently,

$$
\begin{aligned}
2 \int_{a}^{b} y y^{\prime} P_{1} & <\left(\rho^{3}[1-\mu]\right)^{-1}\left(C\left(\mu^{-3}-1\right) \rho^{-1}+\rho^{2} \int_{\mu}^{R} P_{1}+3 \delta / 8\right) \\
& <\left(\rho^{3}[1-\mu]\right)^{-1}\left(\delta / 2+\rho^{2} \int_{\rho}^{R} P_{1}\right) .
\end{aligned}
$$

Hence,

$$
\begin{aligned}
I_{b}(y) & =\int_{a}^{b} r\left(y^{\prime \prime}\right)^{2}+2 y y^{\prime} P_{1} \\
& <\left(\rho^{3}[1-\mu]\right)^{-1}\left(7 \frac{1}{32} M+7 \delta / 8+\delta / 8+\delta / 8+\delta / 2+\rho^{2} \int_{\rho}^{R} P_{1}\right) \leqq 0
\end{aligned}
$$

which completes the proof.

3. The oscillation of $L_{2 n} y=(-1)^{n}\left(r y^{(n)}\right)^{(n)}+p y$.

THEOREM 3.1. If $p(x) \leqq 0,0<r(x) \leqq M x^{\alpha}$ for $\alpha<2 n-1$, and

$$
\lim _{x \rightarrow \infty} \sup x^{2 n-1-\alpha} \int_{x}^{\infty}|p(t)| d t>M A_{n}^{2}
$$

where

$$
A_{n}^{-1}=\sqrt{2 n-1} /[(n-1) !] \sum_{k=1}^{n}(-1)^{k-1}\left(\begin{array}{c}
n-1 \\
k-1
\end{array}\right)(2 n-k)^{-1}
$$

then $L_{2 n} y=(-1)^{n}\left(r y^{(n)}\right)^{(n)}+p(x) y$ is oscillatory on $(0, \infty)$.

Proof. Let $\xi(x)$ be the polynomial of degree $2 n-1$ such that $\xi(0)=\xi^{(k)}(0)=\xi^{(k)}(1)=0$ for $k=1,2, \cdots, n-1$ and $\xi(1)=1$. Given $a>0$, define $y(x)$ as follows:

$$
y(x)= \begin{cases}0 & x<\mu \rho \\ \xi([x-\mu \rho] /[\rho(1-\mu)]) & \mu \rho \leqq x<\rho \\ 1 & \rho \leqq x<R \\ \xi([\nu R-x] /[R(\nu-1)]) & R \leqq x<\nu R \\ 0 & \nu R \leqq x\end{cases}
$$

It can be shown that $\int_{0}^{1}\left(\xi^{(n)}(x)\right)^{2} d x=A_{n}^{2}$.

A result due to Glazman [2, p. 100] considers the case when $\alpha \leqq$ 0 . Consequently, we will consider here only the case in which $\alpha>0$. Since

$$
\begin{aligned}
\int_{\mu \rho}^{\nu R} r\left(y^{(n)}\right)^{2} \leqq & M \rho^{\alpha} \int_{\mu \rho}^{\rho}\left(y^{(n)}\right)^{2}+M(\nu R)^{\alpha} \int_{R}^{\nu R}\left(y^{(n)}\right)^{2} \\
= & M A_{n}^{2} /\left[\rho^{2 n-1-\alpha}(1-\mu)^{2 n-1}\right] \\
& +M A_{n}^{2} /\left[R^{2 n-1-\alpha}\left(\nu^{1-\alpha /(2 n-1)}-\nu^{-\alpha /(2 n-1)}\right)^{2 n-1}\right]
\end{aligned}
$$


and $p(x) \leqq 0$, then

$$
\begin{aligned}
& I_{\nu R}(y)=\int_{\mu \rho}^{R}\left[r\left(y^{(n)}\right)^{2}+p y^{2}\right] \\
& \leqq \frac{1}{\rho^{2 n-1-\alpha}}\left(\frac{M A_{n}^{2}}{(1-\mu)^{2 n-1}}+\frac{M A_{n}^{2} \rho^{2 n-1-\alpha}}{R^{2 n-1-\alpha}\left(\nu^{1-\alpha /(2 n-1)}-\nu^{-\alpha /(2 n-1)}\right)^{2 n-1}}-\rho^{2 n-1-\alpha} \int_{\rho}^{R}|p|\right) .
\end{aligned}
$$

There is a sequence $\left\langle\rho_{k}\right\rangle \rightarrow \infty$ and a number $\delta>0$ such that

$$
\lim _{k \rightarrow \infty} \rho_{k}^{2 n-1-\alpha} \int_{\rho_{k}}^{\infty}|p| \geqq M A_{n}^{2}+\delta .
$$

Choose $\mu>0$ so small that

$$
M A_{n}^{2} /\left[(1-\mu)^{2 n-1}\right]<M A_{n}^{2}+\delta / 4 .
$$

There is a number $K$ so that $\mu \rho_{k}>a$ and

$$
\rho_{k}^{2 n-1-\alpha} \int_{\rho_{k}}^{\infty}|p|>M A_{n}^{2}+3 \delta / 4
$$

for all $k \geqq K$. Set $\rho=\rho_{K}$. Choose $R$ so large that

$$
\rho^{2 n-1-\alpha} \int_{\rho}^{R}|p|>M A_{n}^{2}+\delta / 2
$$

Choose $\nu>1$ so large that

$$
M A_{n}^{2} \rho^{2 n-1-\alpha} /\left[R^{2 n-1-\alpha}\left(\mathcal{\nu}^{1-\alpha /(2 n-1)}-\mathcal{\nu}^{-\alpha /(2 n-1)}\right)^{2 n-1}\right]<\delta / 4 .
$$

We now have that $I_{\nu R}(y)<0$ which implies that $L_{2 n}$ is oscillatory on $(0, \infty)$.

THEOREM 3.2. If there are numbers $M$ and $\alpha$ such that $0<r(x) \leqq$ $M x^{\alpha}$ and if for some $\nu>1$ and $A_{n}$ as in Theorem 3.1

$$
\lim _{x \rightarrow \infty}\left(K x^{\alpha-2 n+1}+\int_{a}^{x} p\right)=-\infty
$$

where $K=M A_{n}^{2} \nu^{\alpha} /(\nu-1)^{2 n-1}$ then $L_{2 n} y=(-1)^{n}\left(r y^{(n)}\right)+$ py is oscillatory on $(0, \infty)$.

Proof. For $\mu, \rho, R$, and $\nu$ below, let $y(x)$ be as in the proof of Theorem 3.1. Pick $\mu$ and $\nu$ so that $0<\mu<1$ and $\nu>1$. Pick $\rho$ so large that $\mu \rho \geqq a$. As in the proof of Theorem 3.1

$$
\int_{\mu \rho}^{\nu R} r\left(y^{(n)}\right)^{2} \leqq M A_{n}^{2}\left(\frac{\rho^{\alpha-2 n+1}}{(1-\mu)^{2 n-1}}+\frac{R^{\alpha-2 n+1} \nu^{\alpha}}{(\nu-1)^{2 n-1}}\right) .
$$

There is a number $c$ such that 


$$
M A_{n}^{2}\left(\frac{\rho^{\alpha-2 n+1}}{(1-\mu)^{2 n-1}}+\frac{x^{\alpha-2 n+1} \nu^{\alpha}}{(\nu-1)^{2 n-1}}\right)+\int_{\mu \rho}^{\rho} p y^{2}+\int_{\rho}^{x} p<0
$$

for all $x \geqq c$. Let $T(x)=\int_{c}^{x} p$. Since $T(x) \rightarrow-\infty$ as $x \rightarrow \infty$, there is a last zero of $T(x)$. Let $R$ be the last zero of $T(x)$. This implies that

$$
\int_{R}^{\nu R} p y^{2}=-2 \int_{R}^{\nu R} y y^{\prime} T(x)<0
$$

Since

$$
\int_{\mu \rho}^{\nu R} p y^{2}<\int_{\mu \rho}^{\rho} p y^{2}+\int_{\rho}^{R} p
$$

then $I_{R}(y)<0$.

\section{REFERENCES}

1. J. H. Barrett, Oscillation theory of ordinary linear differential equations, Advances in Mathematics, 3 (1969), 415-509.

2. I. M. Glazman, Direct Methods of Qualitative Spectral Analysis of Singular Differential Operators, Israel Program for Scientific Translation, Jerusalem, 1965.

3. E. Hille, Nonoscillation theorems, Trans. Amer. Math. Soc., 64 (1948), 234-252.

4. D. B. Hinton, Clamped end boundary conditions for fourth-order self-adjoint differential equations, Duke Math. J., 34 (1967), 131-138.

5 . - A criterion for $(n, n)$ oscillation in differential equations of order $2 n$, Proc. Amer. Math. Soc., 19 (1968), 511-518.

6. R. W. Hunt, The behavior of solutions of ordinary self-adjoint differential equations of arbitrary even order, Pacific J. Math., 12 (1962), 945-961.

7. R. W. Hunt and M. S. T. Namboodiri, Solution behavior for general self-adjoint differential equations, Proc. London Math. Soc., (3) 21 (1970), 637-50.

8. W. Leighton and Z. Nehari, On the oscillation of solutions of self-adjoint differential equations of the fourth order, Trans. Amer. Math. Soc., 89 (1958), 325-377.

9. W. T. Reid, Riccati matrix differential equations and nonoscillation criteria for associated linear systems, Pacific J. Math., 13 (1963), 665-685.

Received December 18, 1972 and in revised form June 15, 1973.

UNIVERSITy OF TENNESSEe at KNOXVILLE

AND

Slippery Rock State College 


\section{PACIFIC JOURNAL OF MATHEMATICS}

\section{EDITORS}

RICHARD ARENS (Managing Editor)

University of California

Los Angeles, California 90024

R. A. BeAumont

University of Washington

Seattle, Washington 98105
J. DugundJI*

Department of Mathematics

University of Southern California

Los Angeles, California 90007

D. Gilbarg and J. Milgram

Stanford University

Stanford, California 94305

\section{ASSOCIATE EDITORS}

E. F. BECKENBACH

B. H. NEUMANN

F. WOLF

K. YoSHIDA

\section{SUPPORTING INSTITUTIONS}

UNIVERSITY OF BRITISH COLUMBIA
CALIFORNIA INSTITUTE OF TECHNOLOGY
UNIVERSITY OF CALIFORNIA
MONTANA STATE UNIVERSITY
UNIVERSITY OF NEVADA
NEW MEXICO STATE UNIVERSITY
OREGON STATE UNIVERSITY
UNIVERSITY OF OREGON
OSAKA UNIVERSITY

UNIVERSITY OF BRITISH COLUMBIA CALIFORNIA INSTITUTE OF TECHNOLOGY UNIVERSITY OF CALIFORNIA MONTANA STATE UNIVERSITY NEW MEXICO STATE UNIVERSITY UNIVERSITY OF OREGON OSAKA UNIVERSITY
UNIVERSITY OF SOUTHERN CALIFORNIA STANFORD UNIVERSITY UNIVERSITY OF TOKYO UNIVERSITY OF UTAH WASHINGTON STATE UNIVERSITY UNIVERSITY OF WASHINGTON AMERICAN MATHEMATICAL SOCIETY NAVAL WEAPONS CENTER

* C. R. DePrima California Institute of Technology, Pasadena, CA 91109, will replace J. Dugundji until August 1974. 


\section{Pacific Journal of Mathematics}

\section{Vol. 51, No. $1 \quad$ November, 1974}

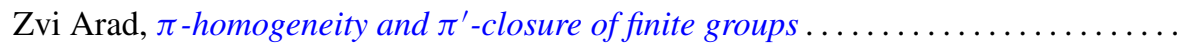

Ivan Baggs, A connected Hausdorff space which is not contained in a maximal

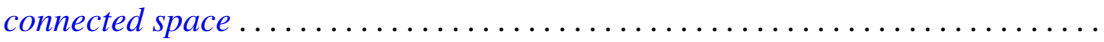

Eric Bedford, The Dirichlet problem for some overdetermined systems on the unit ball in $C^{n}$

R. H. Bing, Woodrow Wilson Bledsoe and R. Daniel Mauldin, Sets generated by

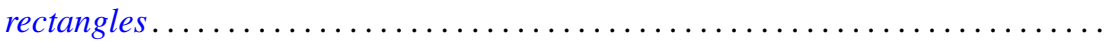

Carlo Cecchini and Alessandro Figà-Talamanca, Projections of uniqueness for

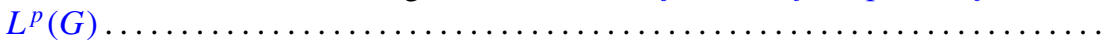

Gokulananda Das and Ram N. Mohapatra, The non absolute Nörlund summability of Fourier series .

Frank Rimi DeMeyer, On separable polynomials over a commutative ring ........ Richard Detmer, Sets which are tame in arcs in $E^{3} \ldots \ldots \ldots \ldots \ldots \ldots \ldots \ldots$

William Erb Dietrich, Ideals in convolution algebras on Abelian groups ..........

Bryce L. Elkins, A Galois theory for linear topological rings .................

William Alan Feldman, A characterization of the topology of compact convergence on $C(X)$.

Hillel Halkin Gershenson, A problem in compact Lie groups and framed cobordism

Samuel R. Gordon, Associators in simple algebras.

Marvin J. Greenberg, Strictly local solutions of Diophantine equations

Jon Craig Helton, Product integrals and inverses in normed rings . . . . . . . . . . . .

Domingo Antonio Herrero, Inner functions under uniform topology . . .

Jerry Alan Johnson, Lipschitz spaces .

Marvin Stanford Keener, Oscillatory solutions and multi-point boundary value

functions for certain nth-order linear ordinary differential equations.

John Cronan Kieffer, A simple proof of the Moy-Perez generalization of the

Shannon-McMillan theorem .......................

Joong Ho Kim, Power invariant rings

Gangaram S. Ladde and V. Lakshmikantham, On flow-invariant sets .

Roger T. Lewis, Oscillation and nonoscillation criteria for some self-adjoint even

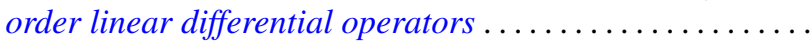

Jürg Thomas Marti, On the existence of support points of solid convex sets ..

John Rowlay Martin, Determining knot types from diagrams of knots . .

James Jerome Metzger, Local ideals in a topological algebra of entire functions

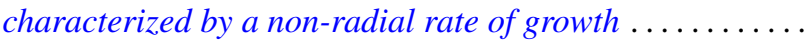

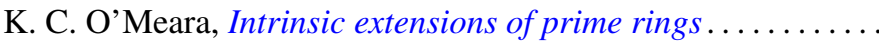

Stanley Poreda, A note on the continuity of best polynomial approximations ..

Robert John Sacker, Asymptotic approach to periodic orbits and local prolongations

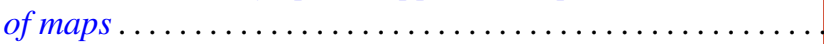

Eric Peter Smith, The Garabedian function of an arbitrary compact set . .

Arne Stray, Pointwise bounded approximation by functions satisfying a side condition

John St. Clair Werth, Jr., Maximal pure subgroups of torsion complete abelian

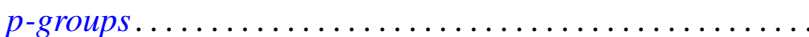

